\title{
Mechanics of a discrete chain with bi-stable elements
}

\author{
G. Puglisi ${ }^{\mathrm{a}}$, L. Truskinovsky ${ }^{\mathrm{b}, *}$ \\ ${ }^{a}$ Dipartimento Ingegneria Strutturale, Politecnico di Bari, Bari, Italy \\ ${ }^{\mathrm{b}}$ Department of Aerospace Engineering and Mechanics, University of Minnesota, MN 55455, USA
}

Received 5 October 1998

\begin{abstract}
It has become common to model materials supporting several crystallographic phases as elastic continua with non (quasi) convex energy. This peculiar property of the energy originates from the multi-stability of the system at the microlevel associated with the possibility of several energetically equivalent arrangements of atoms in crystal lattices. In this paper we study the simplest prototypical discrete system - a one-dimensional chain with a finite number of bi-stable elastic elements.

Our main assumption is that the energy of a single spring has two convex wells separated by a spinodal region where the energy is concave. We neglect the interaction beyond nearest neighbors and explore in some detail a complicated energy landscape for this mechanical system. In particular we show that under generic loading the chain possesses a large number of metastable configurations which may contain up to one (snap) spring in the unstable (spinodal) state. As the loading parameters vary, the system undergoes a number of bifurcations and we show that the type of a bifurcation may depend crucially on the details of the concave (spinodal) part of the energy function. In special cases we obtain explicit formulas for the local and global minima and provide a quantitative description of the possible quasi-static evolution paths and of the associated hysteresis. (C) 1999 Elsevier Science Ltd. All rights reserved.
\end{abstract}

Keywords: A. Phase transformations; Twinning; B. Crystal plasticity; C. Hysteresis

\footnotetext{
* Corresponding author.
}

0022-5096/00/\$ - see front matter (C) 1999 Elsevier Science Ltd. All rights reserved.

PII: S0022-5096(00)00006-X 


\section{Introduction}

Technological advances in the new electronic and biomedical systems increasingly rely on the development of new materials that can actively respond to environmental changes and provide functions such as sensing, processing, actuation and feedback. In the past, 'active' functioning, like sensing and actuation, was achieved through the use of materials characterized by a linear or almost linear response at the microlevel; the complexity of the device was due to the organization of these simple elements into a structure with multiple equilibrium states. Today thin-film micromachinery operates on the parts of a micron and even nanometer size and the traditional solutions are unacceptable; the complexity of the behavior at this scale can only be achieved if a multi-stable 'mechanism' is integrated into the material at the molecular level.

The simplest multi-stable molecular-level mechanism is provided by a crystal phase transition that can be controlled by stress, temperature, electric or magnetic fields. As a result of multi-stability, neighboring constitutive elements may simultaneously occupy different equilibrium states, which leads to highly inhomogeneous equilibrium configurations for the whole body. In particular, one observes a characteristic microstructure which is represented by a large number of plane defects-phase, twin or domain boundaries; the large strains observed in active materials are produced by the coordinated migration of these mobile interfaces in the process of domain 'switching'. The arrangement of elements depends in a crucial way on the history of loading. Because of the nontrivial energetic exchange between the micro and macro levels, multi-stable materials exhibit the capacity to hysteretically recover significant deformation with a controllable amount of energy absorbed in the process.

The main difference between the multi-stable and conventional, linear elements can be traced to the fact that elastic energy of the former is non-convex. After the pioneering work of Ericksen (1975) it has become clear that the non-convexity of the elastic energy is of fundamental importance for the hysteretic behavior of the materials undergoing martensitic phase transformations. Although the recent adaptation of classical theory of nonlinear thermoelasticity for active materials has helped to clarify many important aspects of the formation and evolution of the microstructures, the generic illposedness of the corresponding continuum problems and the multiplicity of available regularization schemes suggest the necessity to study directly the mechanical behavior of the discrete models with multi-stability placed at the microlevel. A deeper understanding of the relation between discrete systems with multi-stable elements and continuum systems with nonconvex energies is also crucial for the development of efficient numerical methods and control codes for active devices.

The discreteness of the system can be viewed as taking place at the level of the atomic lattice (crystal structure) or at some meso-scopic level, where it can be associated with the presence of defects, dislocations, polycrystalline grains, etc. In fact, several levels of discreteness may be of importance. In the situation when the energy of the discrete element is non-convex, the relation between the micro and 
the macro (continuum) models becomes nontrivial. Thus, for instance, the discrete problem presents significantly more local minima than its straightforward 'long wave' approximation; the 'bumpiness' of the energy landscape is effectively smoothed out in the corresponding continuum limit. As a result, the evolution of the discrete system may depend on such factors as 'lattice locking' or 'pinning' and generically leads to a hysteretic behavior (Rogers and Truskinovsky, 1997).

The main goal of this paper is to study the energy landscape for the simplest discrete system with a double-well energy. The approach can be traced back to the work of Muller and Villaggio (1977), who discretized the 1-D continuum problem considered by Ericksen (1975), presented the 'elastic bar' as a chain of bi-stable elements, and for the first time demonstrated numerically the nonuniqueness of metastable equilibria. Later, Fedelich and Zanzotto (1992) re-examined the problem by considering a bi-linear approximation for the force-elongation relation which allowed them to present the complete set of equilibrium configurations in the analytical form and assess their stability (see also Muller and Seelecke, 1996). The bi-linear chain was studied further by Rogers and Truskinovsky (1997) who added an interaction beyond the nearest-neighbors and provided a comparison with the corresponding nonlocal continuum model (Ericksen-Timoshenko bar). Outside the area of phase transitions, a model of a bi-linear chain was employed by Allinger et al. (1996) in simulation of the softening behavior of sarcomers composing the fibers of skeletal muscles. In the context of fracture mechanics, a discrete chain with non-convex springs of the Lennard-Jones type was used by Truskinovsky (1996) and Braides et al. (1998) in a study of a non Cauchy-Born behavior of brittle solids in tension.

The important drawback of most of the above models is the neglect of the spinodal region - a segment of the force-elongation curve with the negative elasticity. Although the wide use of the 'spinodal-free' models is based on the belief that in metastable configurations all elements stay inside the convex energy wells, one cannot remove a priori a possibility that the individual unstable elements are stabilized by the surrounding elements with positive elasticity (Truskinovsky, 1996). A correct description of the spinodal states is also crucial for the evaluation of the energy barriers separating metastable configurations, and for the discussion of a wide spectrum of issues related to the quasistatic evolution, bifurcations and hysteresis.

With this focus on the role of the spinodal region, we study in this paper two representative bi-stable chains with cubic and tri-linear force-elongation relations. The energy of the cubic spring is described by a fourth order polynomial. In the tri-linear model the energy function consists of two upward parabolic wells, which are separated by a spinodal region, where the energy is represented by a downward parabola. This last model allows one to vary the magnitude of the negative elastic modulus inside the spinodal region, is amenable to detailed analytical study and reveals most of the important features of the fully nonlinear model with smooth energy. The tri-linear approximation has been used recently in the corresponding continuum problem by Vainshtein et al. (1998). In the discrete context the tri-linear model was introduced in the paper of Puglisi and 
Truskinovsky (1997), where some preliminary results of the present work have been announced.

The outline of the present paper is as follows. In section 1 we introduce the discrete model, formulate the mathematical problem for the chain in the hard and soft devices and discuss the possible choices for the energy function of a single spring. In section 2 we study the equilibrium configurations and their bifurcations. We construct a detailed bifurcational diagram for the chains with two and three bi-stable springs and provide numerical results for the chains with larger number of elements. The comparison of the chains with cubic and tri-linear springs shows that the latter model distorts the equilibrium solution only in the vicinity of the bifurcation points. In section 3 we study the stability of the equilibrium states and distinguish metastable configurations among the saddle points and local maxima. We conclude with discussion of the main results of the paper.

\section{The model}

\subsection{Mathematical problem}

Consider the equilibrium of a 1-D chain which contains $N$ identical nonlinear elastic springs connected in series. Introduce the undeformed length $a$ and the elongation $\Delta u_{i}=u_{i}-u_{i-1}$ of the $i$-th spring. Suppose $u_{0}=0, u_{N}=\Delta$. The total elastic energy of the chain can be written as

$$
W=\sum_{i=1}^{N} a w\left(\epsilon_{\mathrm{i}}\right)
$$

where

$$
\epsilon_{i}=\Delta u_{i} / a
$$

is the strain in the $i$-th spring. Now, since the total elongation of the chain $\Delta$ is the sum of the elongations $\Delta u_{i}$ of the individual springs, we obtain

$$
\bar{\epsilon}=N^{-1} \sum_{i=1}^{N} \epsilon_{i}
$$

where $\bar{\epsilon}=\Delta /(N a)$ is the total (or average) strain.

If the chain is loaded in a hard device the equilibrium problem reduces to the constrained minimization of the total elastic energy

$$
\left\{\epsilon_{i}, \sum_{i=1}^{N} \epsilon_{i}=N \bar{\epsilon}\right\} \sum^{N}\left(\epsilon_{i}\right) .
$$


In the case of a soft device with the force $\bar{\sigma}$ prescribed, one has to minimize the potential energy

$$
\min _{\left\{\epsilon_{i}\right\}}\left(\sum_{i=1}^{N}\left(w\left(\epsilon_{i}\right)-\bar{\sigma} \epsilon_{i}\right)\right)
$$

If the function $w(\epsilon)$ is convex the solutions of problems (2.3) and (2.4) are trivial, in the sense that the elongations of the springs are identical. This obvious result constitutes the basis of the continuum approximation for the elastic crystals and is often formulated as the Cauchy-Born rule - a statement that the deformation is microscopically homogeneous (Ericksen, 1984). In this paper we will be concerned with the situation when the function $w(\epsilon)$ is non-convex and the corresponding force-strain relation $w^{\prime}(\epsilon)$ is non-monotone. In this case, in addition to the trivial, Cauchy-Born solution, both problems (2.3) and (2.4) allow for a variety of nontrivial equilibrium configurations characterized by the inhomogeneous strain distribution.
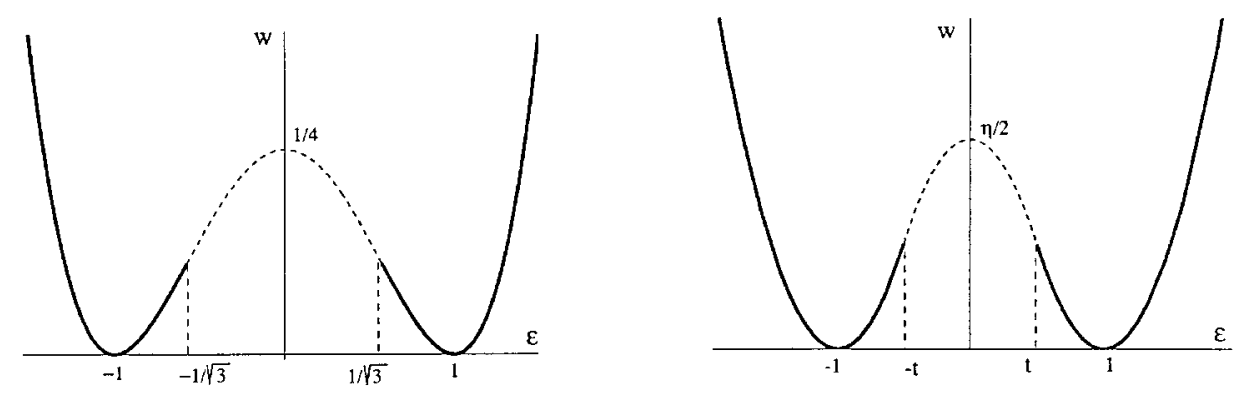

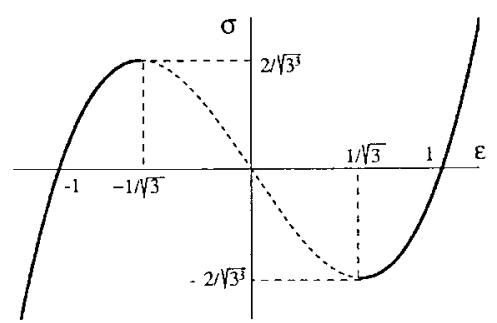

(a)

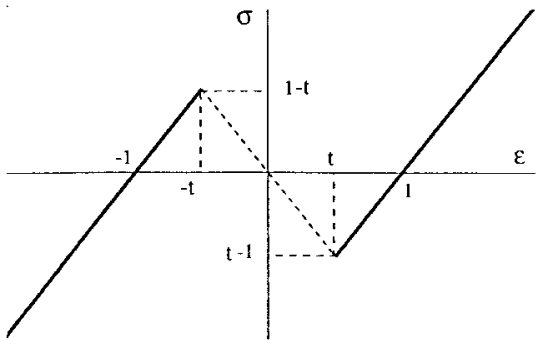

(b)

Fig. 1. Energy-strain $w(\epsilon)$ and force-strain $\sigma(\epsilon)$ relations for a single bi-stable spring: (a) cubic (b) trilinear. Spinodal region: $(-1 / \sqrt{3}, 1 / \sqrt{3})$ in the case of a cubic spring and $(-t, t)$ in the case of a trilinear spring. Stable states are shown with bold lines, unstable states with dashed lines. 


\subsection{Energy}

Consider a 'double-well' energy $w(\epsilon)$ and assume that it is convex in two disjoint intervals. The intervals of convexity (wells) are separated by the spinodal region-an interval where the energy is concave. Under a fixed load an individual spring with this energy can be found in up to three equilibrium states, two of which are stable (bi-stability).

To be specific, consider a class of 'double-well' energies of the type

$$
\bar{w}(\epsilon)=c_{1} w\left(\epsilon / c_{2}\right)+c_{3} \epsilon+c_{4}, c_{i} \geq 0, \quad i=1 \ldots 4,
$$

where

$$
w(\epsilon)=\frac{1}{4}\left(\epsilon^{2}-1\right)^{2} .
$$

In this case the force-strain relation $\sigma=\tilde{\mathrm{w}}^{\prime}(\epsilon)$ is given by a cubic polynomial so we call the corresponding spring cubic; without loss of generality, we shall be using expression (2.6) directly. To study the role of the spinodal region we also consider a 'tri-parabolic' approximation for $w(\epsilon)$

$$
w(\epsilon)=\left\{\begin{array}{cl}
\frac{1}{2}(\epsilon+1)^{2}, & \epsilon<-t \\
\frac{1}{2}\left(\gamma \epsilon^{2}+\eta\right), & -t \leq \epsilon \leq t \\
\frac{1}{2}(\epsilon-1)^{2}, & t<\epsilon
\end{array}\right.
$$

(tri-linear spring). In (2.7) the parameters

$$
\gamma=1-t^{-1}, \quad \eta=1-t
$$

are chosen to make the energy $w(\epsilon)$ smooth (see Fig. 1); the main independent parameter $t \in[0,1]$ characterizes the steepness of the force-strain relation inside the spinodal region. Two special cases of (2.7) deserve special notice: at $t=0$ one obtains a bi-parabolic approximation, and at $t=1$ the energy (2.7) reduces to a convexification of the energy (2.6).

\section{Equilibrium states}

\subsection{Hard device: general case}

Suppose that the average strain $\bar{\epsilon}$ is given and we are interested in finding the strains inside the individual springs. The problem reduces to the study of the critical points for the following function of $N$ variables

$$
W\left(\epsilon_{1}, \ldots \epsilon_{N}\right)=\sum_{i=1}^{N} a w\left(\epsilon_{i}\right),
$$


where the unknowns $\epsilon_{i}, i=1, \ldots, N$ must satisfy

$$
\sum_{i=1}^{N} \epsilon_{i}=N \bar{\epsilon}
$$

The system of equilibrium equations takes the form

$$
w^{\prime}\left(\epsilon_{i}\right)=\sigma, \quad i=1, \ldots, N,
$$

where $\sigma$ is the Lagrange multiplier originating from the constraint (3.2) and representing a constant force in the chain. The total applied force will be denoted by $\bar{\sigma}$, and from (3.3) we obtain that $\sigma=\bar{\sigma}$.

For a bi-stable spring each of the Eqs. (3.3) at a given $\sigma$ may have up to three different solutions, located at the three distinct branches of the inverse function $w^{\prime-1}(\sigma)$. We shall identify these branches as phases I, II, and III. In the case of tri-linear springs (2.7), phase I is defined in the interval $\epsilon<-t$, phase II, in the interval $-t \leq \epsilon \leq t$, and phase III, in the interval $t<\epsilon$; the corresponding strainforce relations can be made explicit

$$
\epsilon_{\mathrm{I}}=\sigma-1, \quad \epsilon_{\mathrm{II}}=\sigma / \gamma, \quad \epsilon_{\mathrm{III}}=\sigma+1 .
$$

In the case of a cubic spring (2.6) the three phases are defined in the intervals $\epsilon<-1 / \sqrt{3},-1 / \sqrt{3} \leq \epsilon \leq 1 / \sqrt{3}$ and $\epsilon>1 / \sqrt{3}$ accordingly and we obtain

$$
\begin{gathered}
\epsilon_{\mathrm{I}}=\frac{\sqrt[3]{3}-1}{\sqrt[3]{4} Q}-\frac{\sqrt[3]{3}+1}{6 \sqrt[3]{2}} Q, \quad \epsilon_{\mathrm{II}}=-\frac{\sqrt[3]{3}+1}{\sqrt[3]{4} Q}-\frac{1-\sqrt[3]{3}}{6 \sqrt[3]{2}} Q, \\
\epsilon_{\mathrm{III}}=\frac{\sqrt[3]{2}}{Q}+\frac{Q}{3 \sqrt[3]{2}},
\end{gathered}
$$

where $Q=27 \sigma \sqrt{\sigma^{2} / 4-1 / 27}$

To parameterize the set of solutions of the system (3.3), introduce three integers $k, l$, $m=0,1, \ldots N$ representing the numbers of springs inside the phases I, II, and III. Parameters $k, l, m$ must satisfy the constraint $k+l+m=N$, where $N$ is the total number of springs. Now, since the total energy $W\left(\epsilon_{1}, \ldots, \epsilon_{N}\right)$ is invariant under the permutation of the indices $i=1, \ldots N$, one can arrange all the solutions of the system (3.3) into isoenergetic equivalence classes, specified by the triples $(k, l, m)$. In addition to the integers $k, l, m$, we shall also introduce the 'volume fractions'

$$
\begin{aligned}
& x=k / N, \quad y=l / N, \quad z=m / N, \\
& x+y+z=1 .
\end{aligned}
$$

The baricentric space of rational triples $(x, y, z)$ will be used as a configurational space; in the continuum limit $a \rightarrow 0, N \rightarrow \infty$ the configurational space corresponds to the space of internal variables.

Given $x, y, z$ and the total load $\bar{\sigma}$, the average strain $\bar{\epsilon}$ can be found from the 
formula

$$
\bar{\epsilon}(\bar{\sigma})=x \epsilon_{\mathrm{I}}(\bar{\sigma})+\gamma \epsilon_{\mathrm{II}}(\bar{\sigma})+z \epsilon_{\mathrm{III}}(\bar{\sigma}),
$$

where the strains $\epsilon_{\mathrm{I}}, \epsilon_{\mathrm{II}}$ and $\epsilon_{\mathrm{III}}$ must be taken from either (3.4) or (3.5). For the tri-linear case (3.5) one can invert (3.7) and obtain the overall force-strain relation

$$
\bar{\sigma}(\bar{\epsilon})=E\left(\bar{\epsilon}-\epsilon_{0}\right),
$$

where

$$
E=\left(1-y \eta^{-1}\right)^{-1}
$$

is the effective elastic modulus along the corresponding branch $(x, y, z)$ and

$$
\epsilon_{0}=z-x
$$

is the reference strain. The relation (3.8) describes a set of linear segments in a $\bar{\sigma}-$ $\bar{\epsilon}$ plane; for a given point in the configurational space, a particular segment $\bar{\sigma}(\bar{\epsilon})$ is defined in the interval with the end points $\bar{\epsilon}^{*}=-t+2 z$ and $\bar{\epsilon}_{*}=t-2 x$. The equilibrium energy of the tri-linear chain is then represented by a set of upward and downward parabolas

$$
\bar{w}(\bar{\epsilon}) \equiv W /(N a)=\frac{1}{2} E\left(\bar{\epsilon}-\epsilon_{0}\right)^{2}+\frac{1}{2} y \eta,
$$

where $\eta$ is given by (2.8). It is easy to check that $\bar{\sigma}=\partial \bar{w} / \partial \bar{\epsilon}$. For the case of cubic springs (3.5), the analogs of formulas (3.8) and (3.11) can also be written explicitly. However, they are too long to be presented here.

Notice that the above set of equilibrium configurations includes local minima, maxima and saddle points. The importance of knowing the energy of the saddle points comes from the fact that the saddles represent the energy barriers between the local minima; Eq. (3.11) and its analog in the case of cubic springs allow one to calculate the activation energy for the corresponding transitions.

To illustrate the general formulas, and to compare the case of cubic springs (2.6) with the tri-linear approximation (2.7), consider in more detail two simple examples.

\subsection{Hard device: $N=2$}

Suppose first, that the chain consists of only two springs so that the unconstrained problem is 2-D. With the average strain fixed, we can eliminate one of the independent variables and introduce an order parameter $\phi$ measuring the deviation from the corresponding Cauchy-Born state

$$
\epsilon_{1}=\bar{\epsilon}-\phi, \quad \epsilon_{2}=\bar{\epsilon}+\phi .
$$

In the new variables, the equilibrium condition reads 


$$
w^{\prime}(\bar{\epsilon}-\phi)=w^{\prime}(\bar{\epsilon}+\phi) \text {. }
$$

We obtain the following equivalence classes of solutions parameterized by the integers $(k, l, m)$ :

- $(2,0,0),(0,2,0),(0,0,2)$ - trivial (Cauchy-Born) branches, corresponding to the homogenous phases I, II, and III;

- $(1,1,0),(1,0,1),(0,1,1)$-nontrivial branches, corresponding to the inhomogeneous microstructures.

The configurational triangle is represented in Fig. 2b.

Consider the case of cubic springs (2.6) first. The trivial branch $\phi=0$ becomes unstable and resumes its stability at the 'spinodal points' $\bar{\epsilon}= \pm 1 / \sqrt{3}$ where $w^{\prime \prime}(\bar{\epsilon})=0$. In the interval $-1 / \sqrt{3} \leq \bar{\epsilon} \leq 1 / \sqrt{3}$ the nontrivial solution includes two symmetric configurations with $\phi= \pm \sqrt{1-3 \bar{\epsilon}^{2}}$. Both bifurcations are pitchfork and energetically equivalent solutions with $\phi>0$ and $\phi<0$ represent symmetry related 'twins'. The bifurcational diagram is presented in Fig. 2a. The graphs of the total energy $\bar{w}(\bar{\epsilon})$ and overall force $\bar{\sigma}(\bar{\epsilon})$ are shown in Fig. 3. One can show that between the two points $A$ and $\alpha$ (points $\beta$ and $B$ ) in Fig. 3 one of the springs assumes a configuration inside the spinodal region and has negative stiffness. This phenomenon of stabilization of an unstable spring by the surrounding stable springs will be discussed in more detail in Section 4.

In the case of tri-linear springs (2.7), the bifurcations leading to the inhomogeneous configurations with $\phi \neq 0$ will be either supercritical for $t>t^{*}=\frac{1}{2}$, or subcritical, for $t<t^{*}=\frac{1}{2}$ (see Fig. 4). To illustrate this point, suppose that we increase the average strain $\bar{\epsilon}$ from the state with $\bar{\epsilon}<-1$ until the

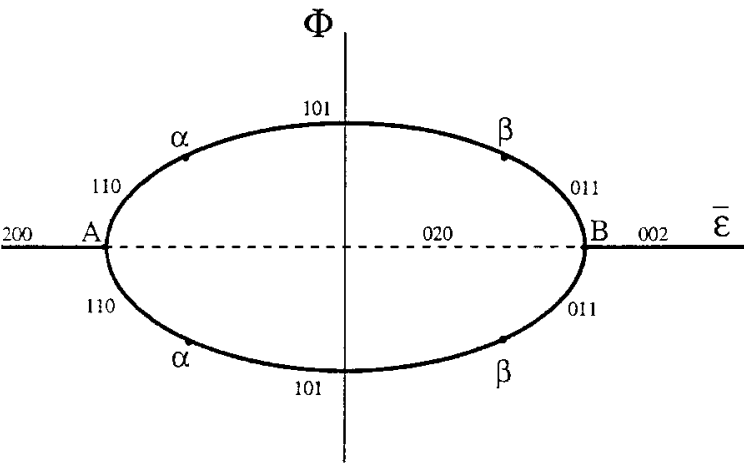

(a)

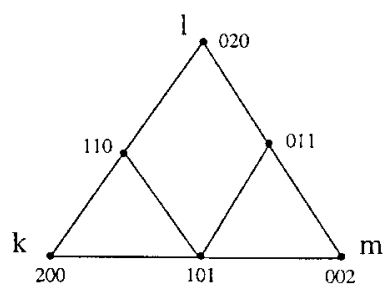

(b)

Fig. 2. (a) Bifurcational diagram and (b) baricentric configurational space for the chain with two cubic springs $(N=2)$. The configuration is indicated by the numbers of springs in different phases. Absolute minima are shown with bold lines, unstable states with dashed lines. Points $A, B, \alpha, \beta$ have the same meaning as in Fig. 3. 

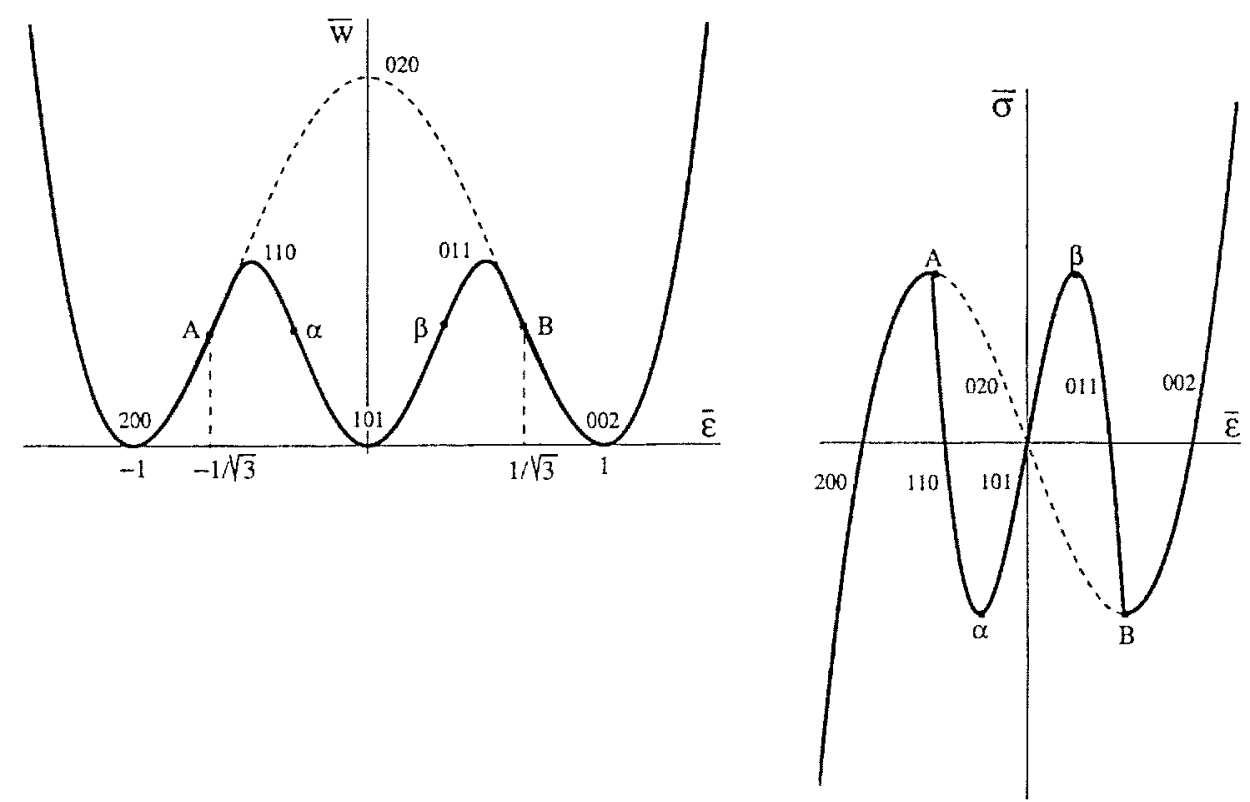

Fig. 3. Overall energy-strain $\bar{w}(\bar{\epsilon})$ and force-strain $\bar{\sigma}(\bar{\epsilon})$ relations for the case $N=2$ (cubic springs). Absolute minima are shown with bold lines, unstable states with dashed lines. Points $A, B, \alpha, \beta$ have the same meaning as in Fig. 2.

trivial configuration $(2,0,0)$ becomes unstable at $\bar{\epsilon}=-t$. The order parameter $\phi$ along the nontrivial branch $(1,1,0)$ (which includes two symmetry related twin configurations with $\epsilon_{1}<\epsilon_{2}$ and $\epsilon_{1}>\epsilon_{2}$ ) can be found from the formulas

$$
\phi= \pm \frac{\bar{\epsilon}+t}{2 t-1} .
$$

Along this branch $y=\frac{1}{2}$, and the overall stiffness equals $E=(t-1) /\left(t-\frac{1}{2}\right)$. We observe that in the supercritical case, the nontrivial minimizer is characterized by a negative overall elasticity. The stability properties of the corresponding configuration in the subcritical case will be clarified in Section 4.

Notice also that the path corresponding to the global minimum of the energy (Maxwell path) shows a qualitatively different pattern in subcritical and supercritical cases. Thus for the case of 'steep' spinodal region $\left(t<t^{*}=\frac{1}{2}\right)$ the energy function along the Maxwell path is non-smooth which results in a discontinuous force elongation curve. In the case of a 'gentle' spinodal region $\left(t>t^{*}=\frac{1}{2}\right)$ the force-strain curve along the Maxwell path is continuous.

\subsection{Hard device: $N=3$}

In the case of three bi-stable springs, the configurational triangle (see Fig. 5 d) shows 10 distinct isoenergetic classes of equilibrium configurations. The natural 

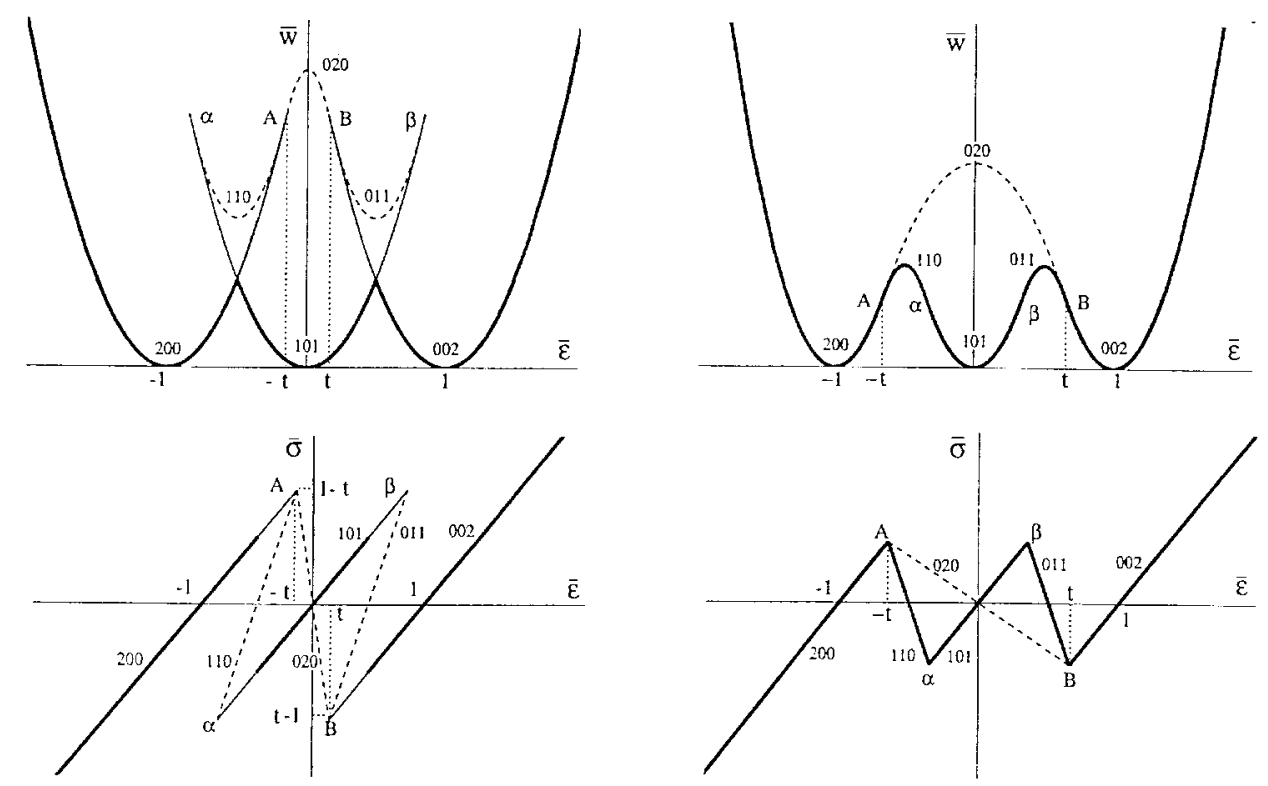

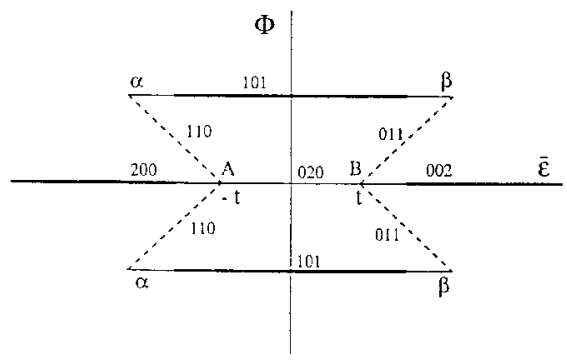

(a)

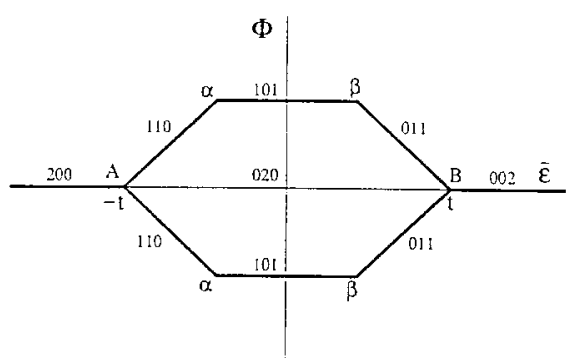

(b)

Fig. 4. Overall energy-strain relation $\bar{w}(\bar{\epsilon})$, force-strain relation $\bar{\sigma}(\bar{\epsilon})$ and bifurcation diagram $\phi(\bar{\epsilon})$ for the case $N=2$ (tri-linear springs): (a) subcritical case $t<t^{*}$; (b) supercritical case $t>t^{*}$. Absolute minima are shown with bold lines, metastable states with solid lines, unstable states with dashed lines. Points $A, B, \alpha, \beta$ have the same meaning as in Figs. 2 and 3.

set of order parameters, which automatically respects the constraint, can be introduced by the formulas

$$
\begin{aligned}
& \epsilon_{1}=\bar{\epsilon}+\phi+\sqrt{3} \psi, \\
& \epsilon_{2}=\bar{\epsilon}+\phi-\sqrt{3} \psi, \\
& \epsilon_{3}=\bar{\epsilon}-2 \phi .
\end{aligned}
$$




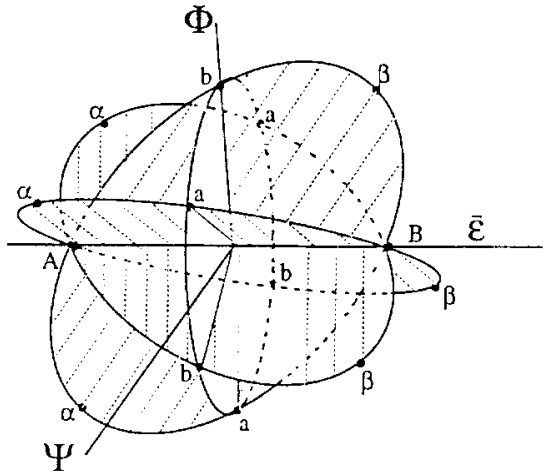

(a)

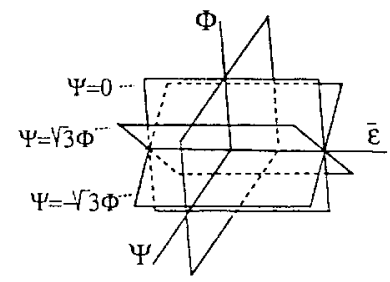

(c)

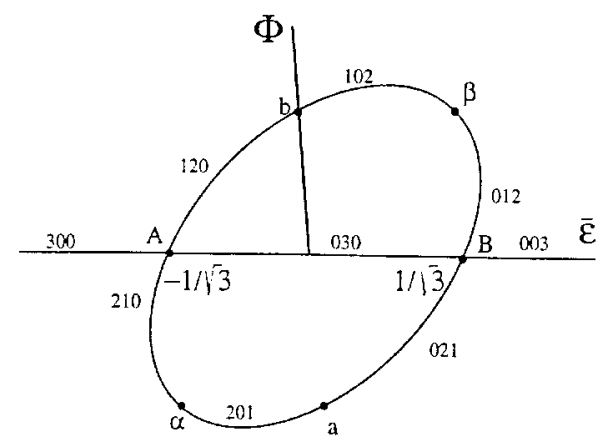

(b)

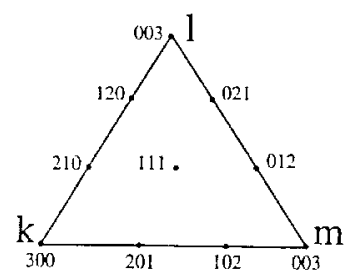

(d)

Fig. 5. (a) Bifurcational diagram and (d) configurational space for the chain with three cubic springs $(N=3)$. A division of the space into tetragonal planes and a particular tetragonal subsection $\psi=0$ of the bifurcational diagram are shown in (c) and (b) accordingly. Points $A, B, \alpha, \beta, a, b$ have the same meaning as in Fig. 6.

In (3.15) the parameter $\phi$ measures the degree of 'non Cauchy-Born' behavior when two springs still have equal strains. The other parameter $\psi$ describes further reduction of symmetry and characterizes the state with all three springs having different strains. In the new variables the system of equilibrium equations takes the form

$$
\begin{aligned}
& w^{\prime}(\bar{\epsilon}+\phi+\sqrt{3} \psi)=w^{\prime}(\bar{\epsilon}+\phi-\sqrt{3} \psi), \\
& w^{\prime}(\bar{\epsilon}+\phi+\sqrt{3} \psi)=w^{\prime}(\bar{\epsilon}-2 \phi) .
\end{aligned}
$$

With the two order parameters $\phi$ and $\psi$ one can represent a complete set of symmetry related equilibrium configurations. Thus, in the space $\phi, \psi$, and $\bar{\epsilon}$, a line $\phi=0, \psi=0$ represents the trivial (Cauchy-Born) branches $(3,0,0),(0,3,0)$ and $(0,0,3)$ while the three symmetric planes $\psi=0, \psi= \pm \sqrt{3} \phi$ contain partially non Cauchy-Born branches $(2,1,0),(2,0,1),(0,2,1),(0,1,2),(1,0,2)$ and $(1,2,0)$. Finally a point on the branch $(1,1,1)$ with all three strains different corresponds to a generic point in the order parameter space with $\phi \neq 0, \psi \neq 0$. 
Remark. There exists an interesting analogy between this problem and the problem of classifying weak phase transitions in Bravais lattices with cubic, tetragonal and orthorhombic symmetries (Ericksen, 1993). This analogy is based on the isomorphism between the two symmetry groups; crystallographic point group in the case of crystals and the group of permutations in the case of springs. With this analogy in mind one can rename our trivial branch - the cubic, the branches with $\psi=0$ and $\psi= \pm \sqrt{3} \phi$ - the tetragonal and the branches with $\phi \neq 0, \psi \neq 0$ the orthorhombic. A generic configuration of a chain may be represented by one cubic configuration, one of the three tetragonal twins or one of the six orthorhombic twins. Below, we shall be using this language for convenience.

The general solution of Eq. (3.17) for a chain with cubic springs is given by the formulas

$$
\begin{aligned}
& \phi=0, \quad \psi=0, \\
& \psi=0, \phi=\frac{\bar{\epsilon}}{2} \pm \sqrt{\frac{1}{3}-\frac{3 \bar{\epsilon}^{2}}{4}} \\
& \psi= \pm \sqrt{3} \phi, \quad \phi=\frac{\bar{\epsilon}}{4} \pm \frac{1}{2} \sqrt{\frac{1}{3}-\frac{3 \bar{\epsilon}^{2}}{4} .}
\end{aligned}
$$
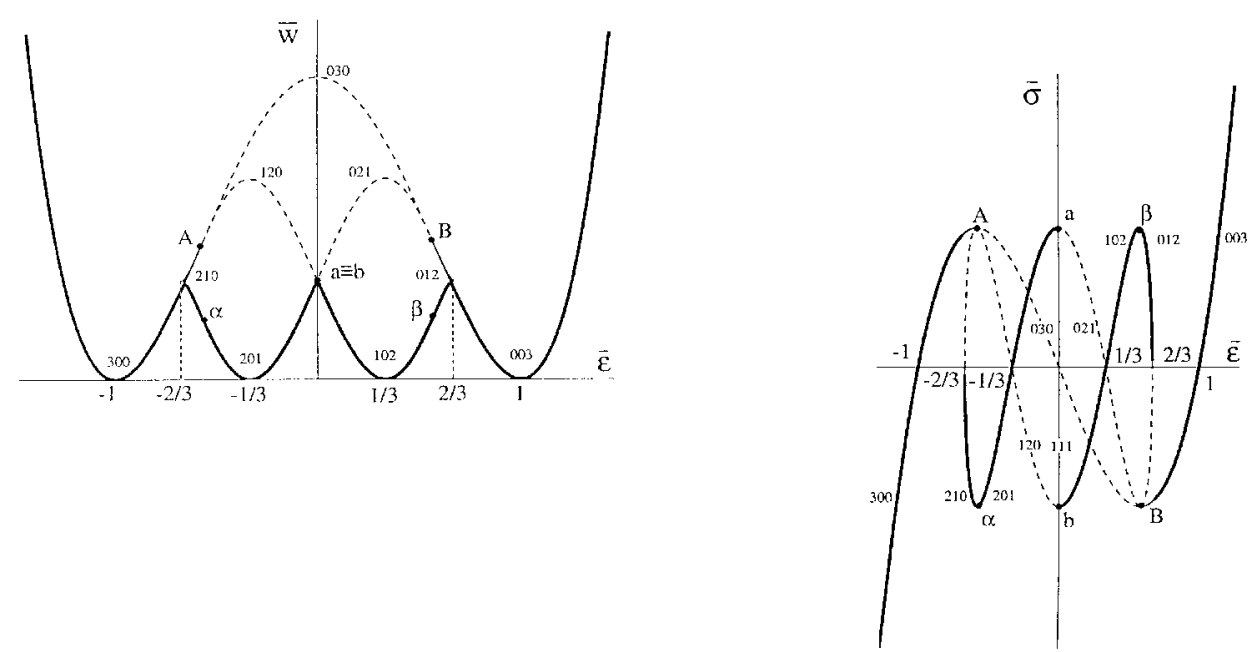

Fig. 6. Overall energy-strain relation $\bar{w}(\bar{\epsilon})$ and force-strain relation $\bar{\sigma}(\bar{\epsilon})$ for the case $N=3$ (cubic springs). Absolute minima are shown with bold lines, metastable states with solid lines, unstable states with dashed lines. Points $A, B, \alpha, \beta, a, b$ have the same meaning as in Fig. 5. 
The complete bifurcational diagram is shown in Fig. 5a. In Fig. 5b we present a subsection $\psi=0$ of the whole bifurcational diagram showing the details of the transition between the cubic phase and one of the tetragonal twins; the orthorhombic branches intersect the plane $\psi=0$ transversely and cannot be seen on this subsection. By using the results from the crystallographic analysis (Ericksen, 1993), we conclude, that the cubic to tetragonal bifurcation, which takes place at
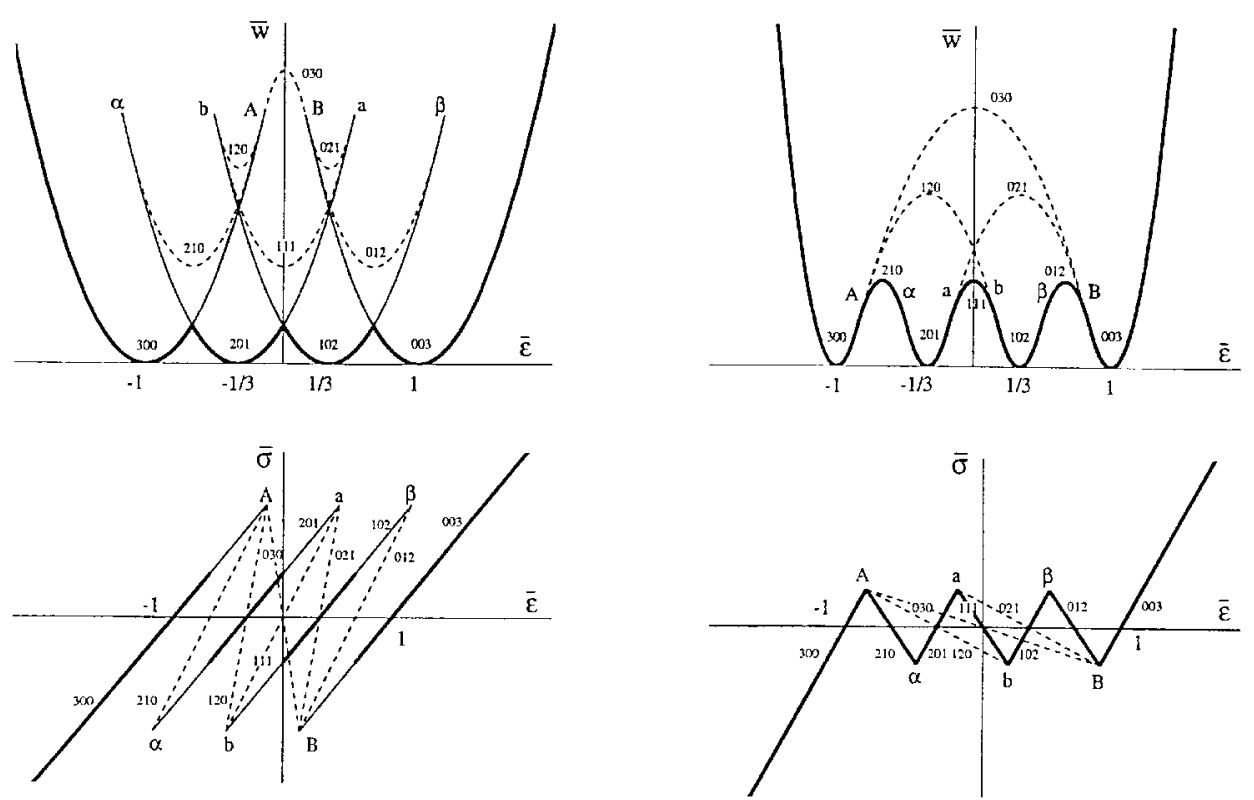

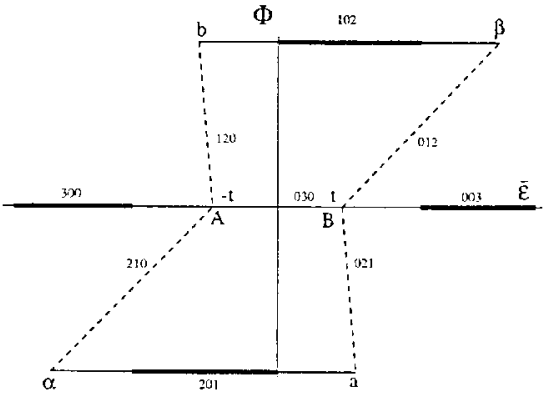

(a)

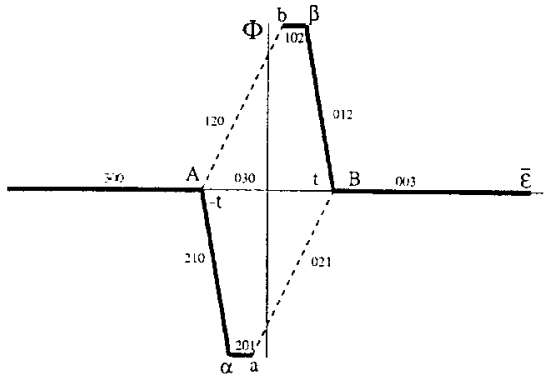

(b)

Fig. 7. Overall energy-strain relation $\bar{w}(\bar{\epsilon})$, force-strain relation $\bar{\sigma}(\bar{\epsilon})$ and a $\psi=0$ subsection of the bifurcation diagram for the case $N=3$ (tri-linear springs): (a) subcritical case $t<t^{*}$; (b) supercritical case $t>t^{*}$. Absolute minima are shown with bold lines, metastable states with solid lines, unstable states with dashed lines. Points $A, B, \alpha, \beta, a, b$ have the same meaning as in Figs. 5 and 6. 
the points where $w^{\prime \prime}(\bar{\epsilon})=0$, is subcritical while the tetragonal to orthorhombic bifurcation, which takes place when $w^{\prime}(\bar{\epsilon}+\phi)=w^{\prime}(\bar{\epsilon}-2 \phi)$ and $w^{\prime \prime}(\bar{\epsilon}+\phi)=0$, is a pitchfork. The three tetragonal branches are represented on the bifurcational diagram by the noncoplanar ellipses located inside the planes $\psi=0, \psi= \pm \sqrt{3} \phi$ (see Fig. 5c), while the orthorhombic configurations confined to the plane $\bar{\epsilon}=0$ form six connecting (out of the planes) segments.

The graphs of the overall energy - strain relation, $\bar{w}(\bar{\epsilon})$, and the overall forcestrain relation, $\bar{\sigma}(\bar{\epsilon})$, are shown in Fig. 6 . Contrary to the case $N=2$, the Maxwell path is represented on the force-strain plane by a discontinuous curve. One can show that this path again contains configurations with one unstable spring.

To illustrate the nature of the tri-linear approximation, we can now compare the above pictures with the exact solution for the energy (2.7). The situation here is similar to what we have already seen in the case $N=2$. Thus, one can show that the bifurcation from the trivial, cubic branch $(3,0,0)$ to the tetragonal branch $(2,1,0)$ at $\bar{\epsilon}=-t$ is subcritical for $t<t^{*}=\frac{2}{3}$. The three symmetry related tetragonal twins of the $(2,1,0)$ equivalence class are given by the formulas

$$
\left\{\begin{array} { c } 
{ \epsilon _ { 1 } = \epsilon _ { 2 } \leq \epsilon _ { 3 } } \\
{ \phi = \frac { \overline { \epsilon } + t } { 2 - 3 t } } \\
{ \psi = 0 }
\end{array} \left\{\begin{array} { l } 
{ \epsilon _ { 1 } = \epsilon _ { 3 } \leq \epsilon _ { 2 } } \\
{ \phi = \frac { \overline { \epsilon } + t } { 2 ( 3 t - 2 ) } } \\
{ \psi = - \sqrt { 3 } \phi }
\end{array} \quad \left\{\begin{array}{l}
\epsilon_{2}=\epsilon_{3} \leq \epsilon_{1} \\
\phi=\frac{\bar{\epsilon}+t}{2(3 t-2)} \\
\psi=\sqrt{3} \phi
\end{array}\right.\right.\right.
$$

The effective elastic modulus for the whole chain along these tetragonal branches $E=(t-1) /\left(t-\frac{2}{3}\right)$ is negative in the supercritical case (when the corresponding branch is globally stable) and is positive in the subcritical case. Both possibilities are illustrated in Fig. 7; notice the transformation of the ellipses from Fig. 5 into hexagons in Fig. 7. The Maxwell path on the force-strain plane is continuous in the supercritical case $t>t^{*}=\frac{2}{3}$ (Fig. 7b) and contains configurations with one spring in the spinodal region. In the subcritical case $\left(t<t^{*}=\frac{2}{3}\right.$, Fig. 7a) the forcestrain Maxwell path is only piece-wise continuous, with no springs in the spinodal region.

\subsection{Hard device: general case}

For larger $N$ the picture soon becomes rather complicated. As we show below in Section 4 the branches without 'spinodal springs' $(l=0)$ and the branches with $l=1$ bifurcating from them are the only candidates as the local minimizers. We first observe that there exist exactly $k-1$ branches of the type $(k-l, l, m)$, $0<l<k$, bifurcating from the branch $(k, 0, m)$. In particular, there are $N-1$ branches bifurcating from the trivial branch $(N, 0,0)$ and only one, bifurcating from the branch $(2,0, N-2)$. These bifurcations are subcritical for $k>2 l$, supercritical for $k<2 l$ and it is a pitchfork for $k=2 l$. The bifurcations leading to the potentially stable branches with only one spring in the spinodal region $(l=1)$ are subcritical for all $k>2$. For example, in case $N=2$, we had a trivial 
branch with $k=2$ and the bifurcating nontrivial branch with $l=1$ was a pitchfork. In the second example, $N=3$, we had a trivial configuration with $k=3$ and the bifurcation leading to the configuration with $l=1$ was subcritical. To illustrate these general conclusions we present in Fig. 8 the overall energystrain and force-strain relations for the chain with six cubic springs $(N=6)$.

Comparison of the cubic model with its tri-linear approximation shows that the overall behavior is modified only around the bifurcation points. In the tri-linear case and general $N$ the critical value of $t$ which separates the case when the bifurcation from the branch $l=0$ to the branch $l=1$ is subcritical from the case when it is supercritical is given by the formula

$$
t^{*}=1-N^{-1} \text {. }
$$

For $t<t^{*}$ the Maxwell path in the configurational space $(x, y, z)$ is located on the edge $y=0$, while for $t>t^{*}$ it is represented by a sawtooth curve approaching, as $N \rightarrow \infty$, a generalized curve. The corresponding path on the force-strain plane is discontinuous in the subcritical case and is continuous in the supercritical case. This behavior is illustrated in Fig. 9 where both subcritical and supercritical cases are presented.
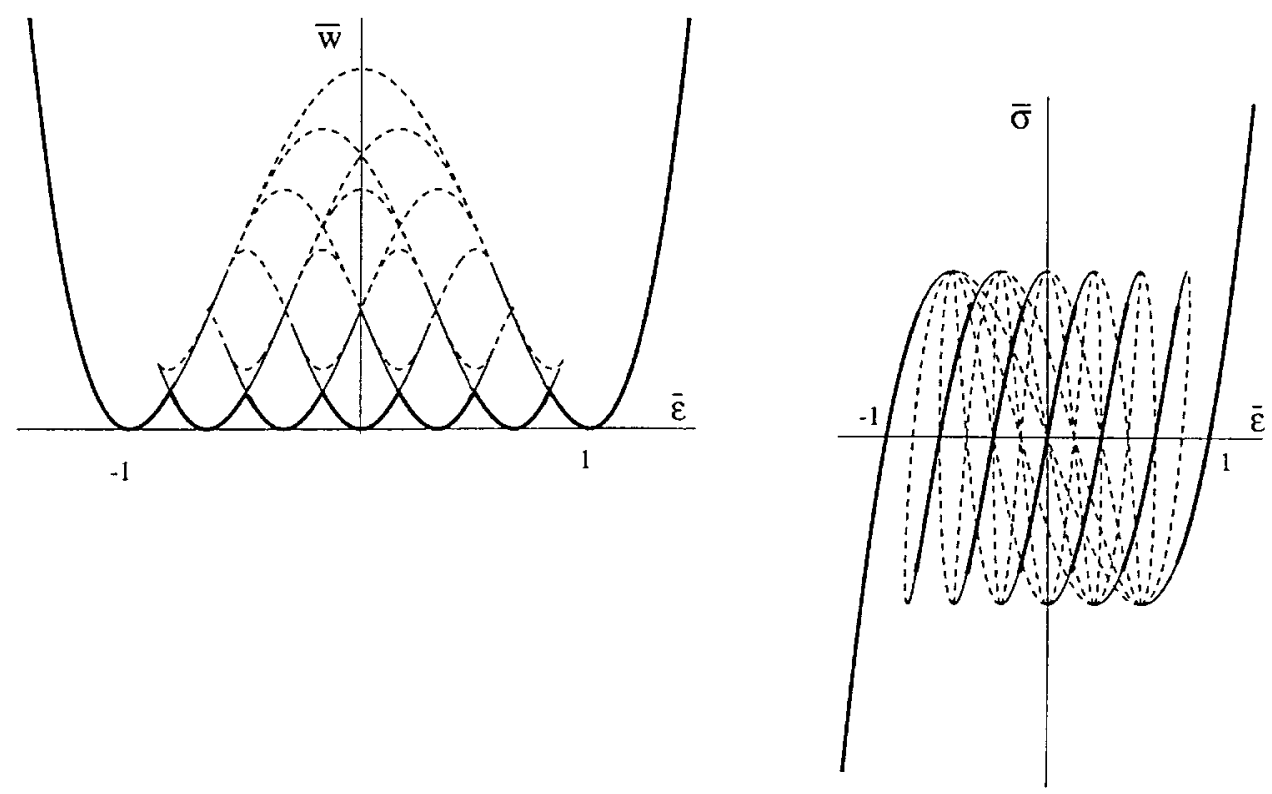

Fig. 8. Overall energy-strain relation $\bar{w}(\bar{\epsilon})$ and force-strain relation $\bar{\sigma}(\bar{\epsilon})$ for the chain with six cubic springs $(N=6)$. Absolute minima are shown with bold lines, metastable states with solid lines, unstable states with dashed lines. 

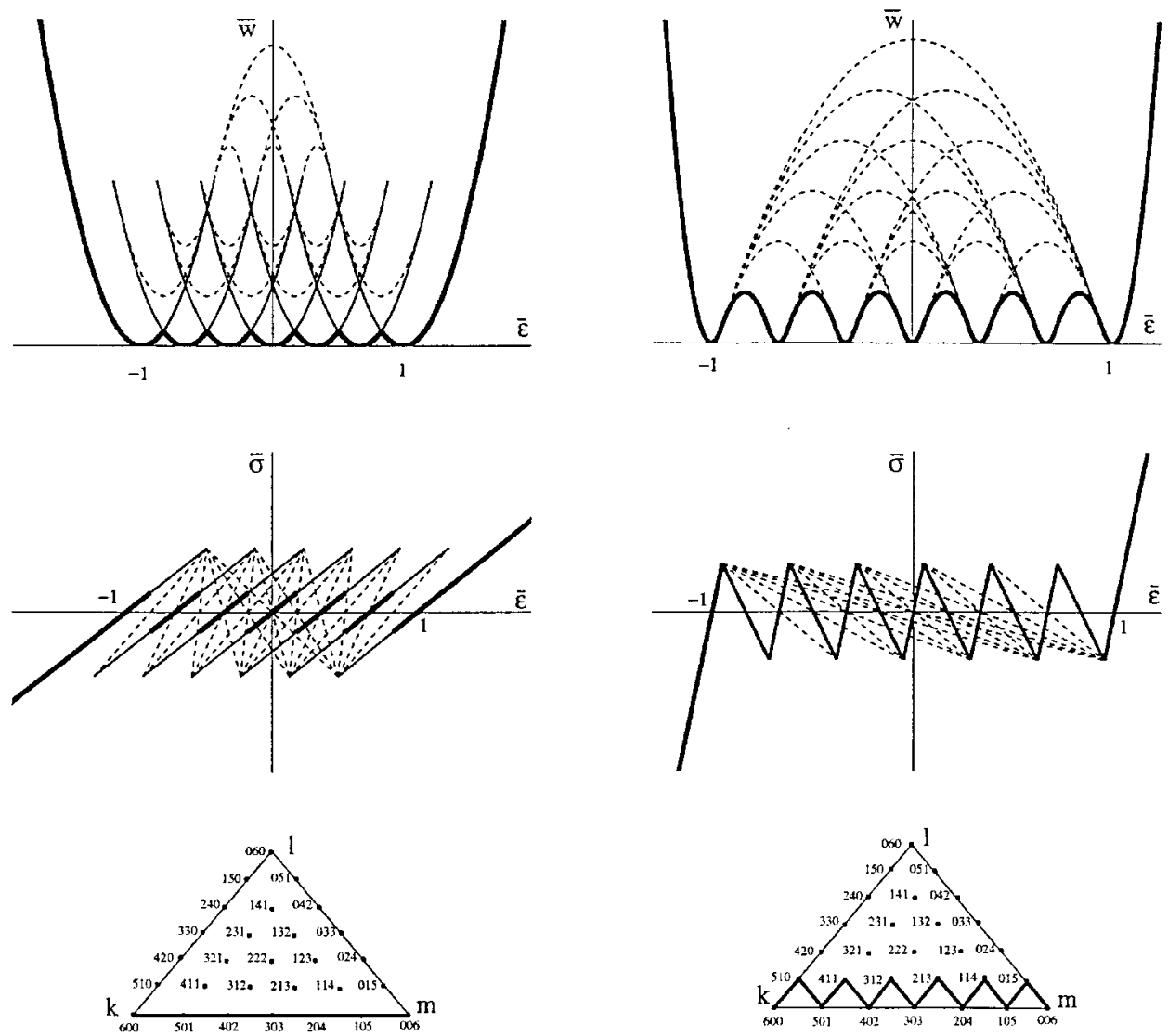

(a)

(b)

Fig. 9. Overall energy-strain relation $\bar{w}(\bar{\epsilon})$, force-strain relation $\bar{\sigma}(\bar{\epsilon})$ and the baricentric configurational space for the case $N=6$ (tri-linear springs): (a) subcritical case $t\left\langle t^{*}\right.$; (b) supercritical case $\left.t\right\rangle t^{*}$. Absolute minima are shown with bold lines, metastable states with solid lines, unstable states with dashed lines.

\subsection{Soft device: general case}

Suppose now that the total force $\bar{\sigma}$ acting on the chain is prescribed. The mathematical problem reduces to the study of the critical points for the total (or Gibbs') energy

$$
G\left(\epsilon_{1}, \ldots \epsilon_{N}\right)=\sum_{i=1}^{N} a\left(w\left(\epsilon_{i}\right)-\bar{\sigma} \epsilon_{i}\right)
$$

The system of equilibrium equations takes the form 


$$
w^{\prime}\left(\epsilon_{i}\right)=\bar{\sigma}, \quad i=1, \ldots, N \text {. }
$$

Since the system (3.22) is identical to (3.3), most of the analysis for the chain in a hard device can be preserved in the case of a soft device. Thus the whole multiplicity of equilibrium solutions can again be arranged into equivalence classes and parameterized by the three integers $k, l, m$ satisfying $k+1+m=N$.

In the special case of tri-linear springs (2.7), the strains in the individual phases are given by the formulas

$$
\epsilon_{\mathrm{I}}=\bar{\sigma}-1, \quad \epsilon_{\mathrm{II}}=\bar{\sigma} / \gamma, \quad \epsilon_{\mathrm{III}}=\bar{\sigma}+1 .
$$

The total strain can now be obtained from

$$
\bar{\epsilon}(\bar{\sigma})=\bar{\sigma} / E+\epsilon_{0},
$$

where the parameters $E$ and $\epsilon_{0}$ are given by (3.9) and (3.10). The nontrivial branches are defined in the interval

$$
t-1 \leq \bar{\sigma} \leq 1-t .
$$

The corresponding energy of the equilibrium chain can then be written as

$$
\bar{g}(\bar{\sigma}) \equiv G /(N a)=-\frac{1}{(2 E)} \bar{\sigma}^{2}-\bar{\sigma} \epsilon_{0}+\frac{1}{2} y \eta .
$$

We recall that again $\bar{\epsilon}=\partial \bar{g} / \partial \bar{\sigma}$. In the case of cubic springs (2.6) the formulas analogous to (2.23) and (2.26) are too long to be presented here.

\subsection{Soft device: illustrations}

Consider the simplest chain with two bi-stable springs $(N=2)$. Introduce two order parameters: $\bar{\epsilon}$ and $\phi$ such that

$$
\epsilon_{1}=\bar{\epsilon}+\phi, \quad \epsilon_{2}=\bar{\epsilon}-\phi .
$$
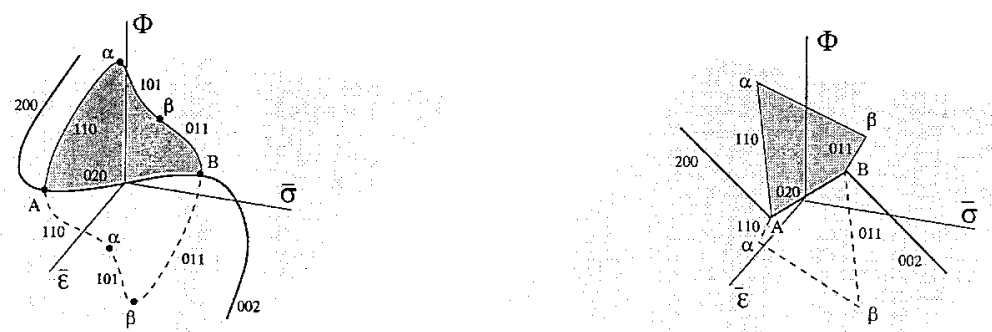

Fig. 10. Bifurcational diagram for the chain with two bi-stable springs $(N=2)$ in a soft device: (a) cubic springs; (b) tri-linear springs. The trivial branches $(\phi=0)$ are shown with bold lines, the nontrivial branches $\phi>0(\phi<0)$-with solid (dashed) lines. Points $A, B, \alpha, \beta$ have the same meaning as in Fig. 11. 
The parameter $\bar{\epsilon}(\bar{\sigma})$ gives the average strain at a given total force $\bar{\sigma}$, and the parameter $\phi(\bar{\sigma})$ measures the deviation from the Cauchy-Born rule. The two symmetry related 'twins' correspond to $\pm \phi(\bar{\sigma})$. For the cubic springs the bifurcational diagram in the space $\phi, \bar{\epsilon}, \bar{\sigma}$ is presented in Fig. 10. The corresponding overall energy-force and strain-force relations are illustrated in Fig. 11; in both figures and diagrams for the tri-linear springs are also presented for comparison. The calculations for a chain with six springs $(N=6)$ are summarized in Fig. 12. Notice that, contrary to the case of hard device, in soft loading the Maxwell (or the global minimum) path does not include any nontrivial (non Cauchy-Born) configurations.
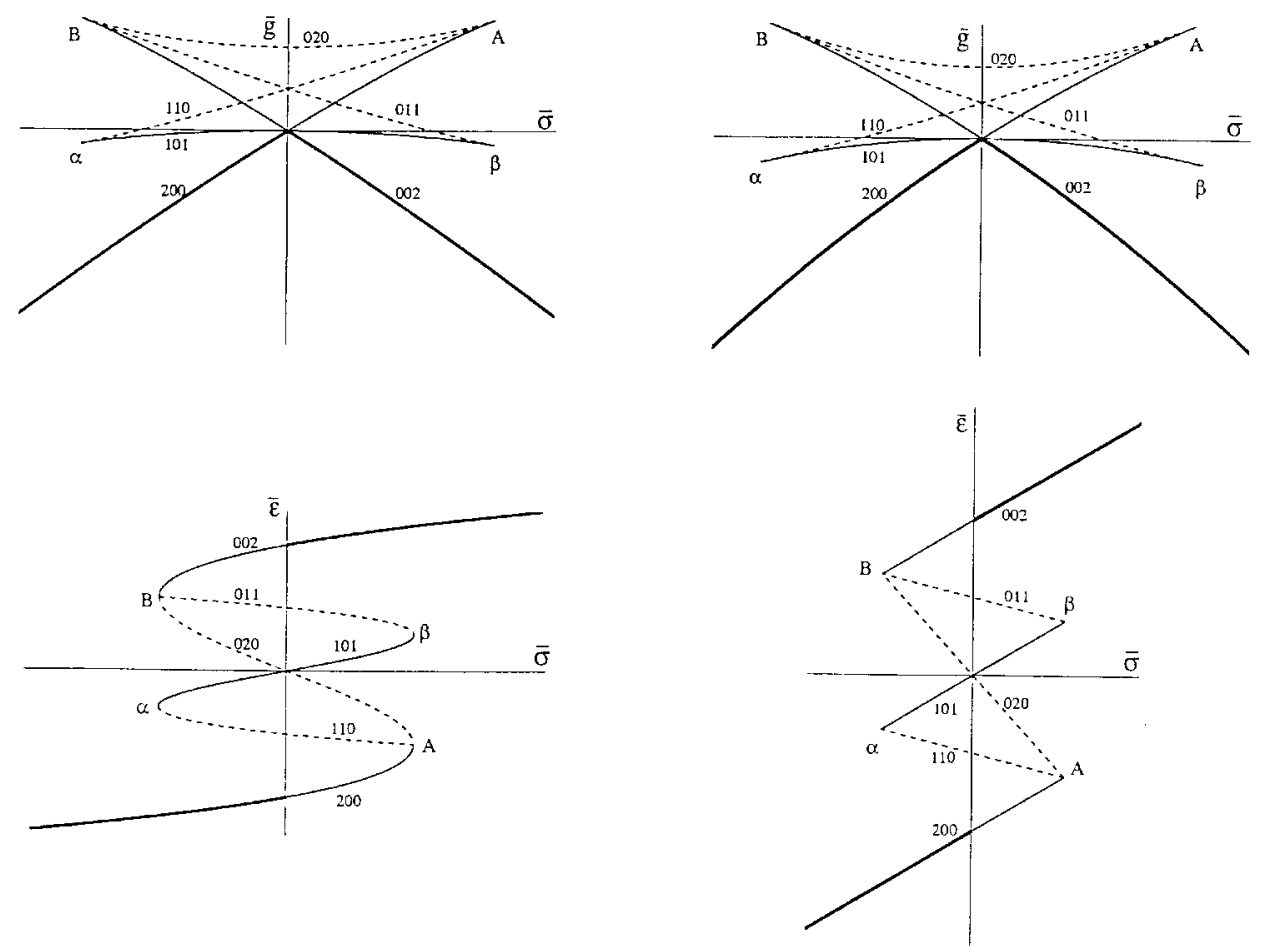

Fig. 11. Overall energy-force relation $\bar{g}(\bar{\sigma})$ and strain-force relation $\bar{\epsilon}(\bar{\sigma})$ for a chain with two springs $(N=2)$ in a soft device: (a) cubic case; (b) tri-linear case. Absolute minima are shown with bold lines, metastable states with solid lines, unstable states with dashed lines. Points $A, B, \alpha, \beta$ have the same meaning as in Fig. 10. 

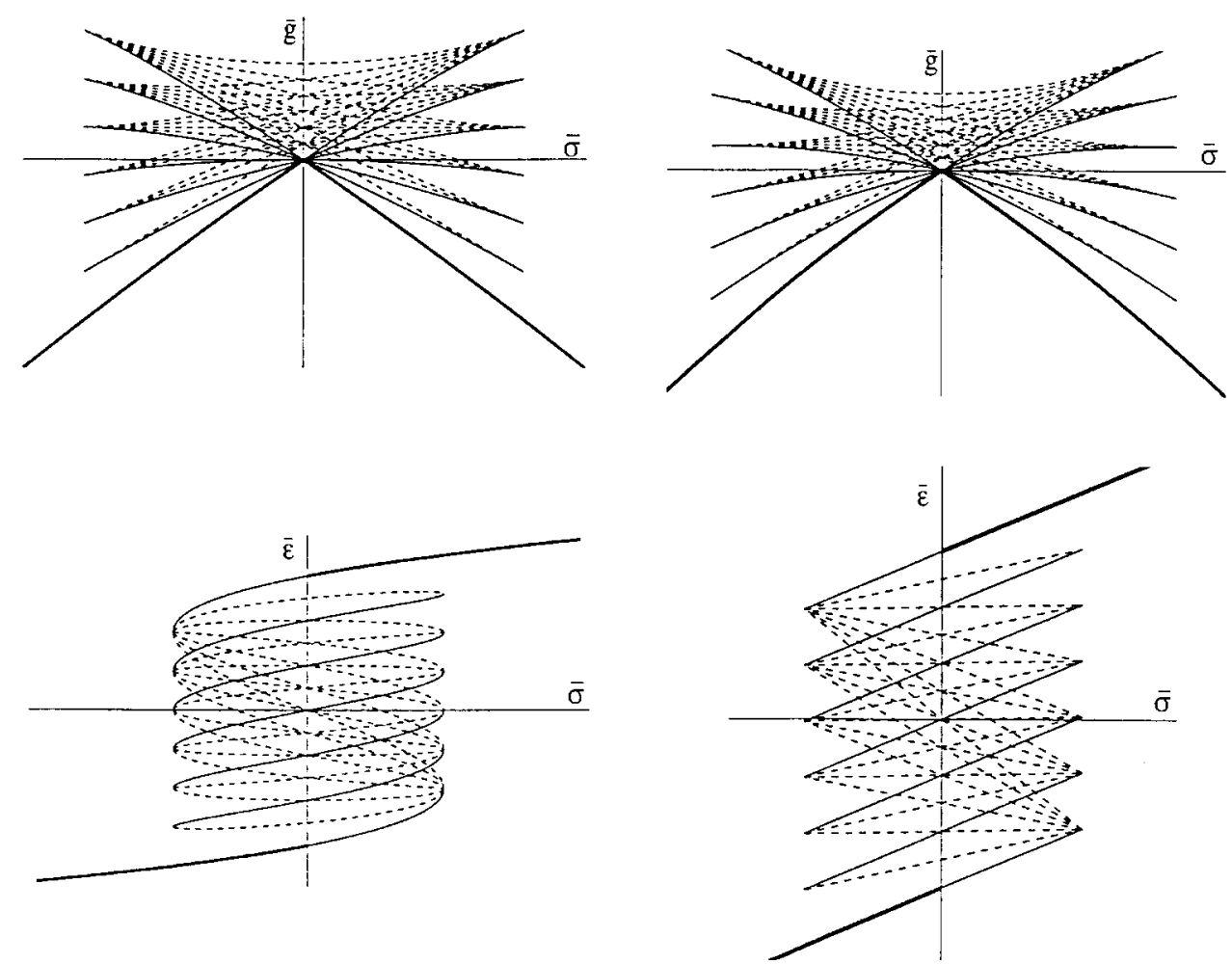

Fig. 12. Overall energy-force relation $\bar{g}(\bar{\sigma})$ and strain-force relation $\bar{\epsilon}(\bar{\sigma})$ for a chain with six springs $(N=6)$ in a soft device: (a) cubic case; (b) tri-linear case. Global minima are shown with bold lines, metastable states with solid lines, unstable states with dashed lines.

\section{Stability}

\subsection{Hard device: general case}

We begin the stability analysis for the chain in a hard device with eliminating the constraint (2.2) and rewriting the energy as a function of $N-1$ variables

$$
\hat{W}\left(\epsilon_{1}, \ldots, \epsilon_{N-1}\right)=W\left(\epsilon_{1}, \ldots, \epsilon_{N-1}, N \bar{\epsilon}-\sum_{i=1}^{N-1} \epsilon_{i}\right) .
$$

In the new variables the Hessian matrix of the second derivatives takes the form

$$
\mathbf{H}=\left|\begin{array}{llll}
E_{1}+E_{N} & E_{N} & \ldots & E_{N} \\
E_{N} & E_{2}+E_{N} & \ldots & E_{N} \\
\ldots & \ldots & \ldots & \ldots \\
E_{N} & E_{N} & \ldots & E_{N-1}+E_{N}
\end{array}\right|,
$$


where $E_{i}=a w^{\prime \prime}\left(\epsilon_{i}\right)$ and the set $\epsilon_{i} i=1, \ldots, N$ corresponds to a particular critical point of (4.1). The equilibrium configuration $\epsilon_{i}(\bar{\epsilon}), i=1, \ldots, N-1$ is a local minimum of the energy $\hat{W}$ if the matrix $\mathbf{H}(\bar{\epsilon})$ is positive definite. To insure the positive definiteness of this matrix, we require

$$
A_{j}>0, \quad j=1, \ldots, N-1,
$$

where

$$
A_{j} \equiv\left(\prod_{i=1}^{j} E_{i}\right)\left(1+\sum_{i=1}^{j} \frac{E_{N}}{E_{i}}\right)
$$

are the principal minors of the matrix (4.2).

The class of equivalent equilibrium solutions is characterized by the three integers $k, l, m$, however the stability properties of the corresponding equilibrium configurations depend only on $l$-the number of springs in the spinodal region. Thus, if $l=0$, we have a branch of the type $k, 0, m$, and all the springs are in the stable states. As a result, we have $E_{i}>0$, for all $i=1, \ldots, N$, and the matrix $\mathbf{H}(\bar{\epsilon})$ is obviously positive definite. Now, if $l>2$, so that the chain contains two or more springs with negative stiffness, one can always regroup the springs in such a way, that the first and the last springs are in the spinodal state. Then $E_{1}<0$ and $E_{N}<0$ and since $A_{1}=E_{1}+E_{N}$ we obtain $A_{1}<0$ which means instability of the corresponding configuration. Finally, if $l=1$, the equilibrium configuration contains exactly one unstable spring and we can always consider that it is the $N-1$ th spring, which is in the spinodal state. As a consequence, $E_{N-1}<0$, while for all other $j=1, \ldots, N-2, N$ we have $E_{j}>0$. Then $A_{j}>0$ for $j=1$, $\ldots, N-2$ and stability depends on the sign of $A_{N-1}$.

For the case $j=N-1$ the general expression (4.4) can be rewritten as

$$
A_{N-1}=\left(\prod_{i=1}^{N} E_{i}\right)\left(\sum_{i=1}^{N} \frac{1}{E_{i}}\right),
$$

or more explicitly as

$$
A_{N-1}=\left(E\left(\epsilon_{\mathrm{I}}\right)\right)^{k}\left(E\left(\epsilon_{\mathrm{II}}\right)\right)^{l}\left(E\left(\epsilon_{\mathrm{III}}\right)\right)^{m}\left(\frac{k}{E\left(\epsilon_{\mathrm{I}}\right)}+\frac{l}{E\left(\epsilon_{\mathrm{II}}\right)}+\frac{m}{E\left(\epsilon_{\mathrm{III}}\right)}\right) .
$$

Now, since $E\left(\epsilon_{\mathrm{II}}\right)<0$, the necessary and sufficient condition for stability yields

$$
\frac{k}{E\left(\epsilon_{\mathrm{I}}\right)}+\frac{1}{E\left(\epsilon_{\mathrm{II}}\right)}+\frac{m}{E\left(\epsilon_{\mathrm{III}}\right)}<0 .
$$

The inequality (4.7) has a simple geometrical interpretation. Since the springs are connected in series we obtain the expression for the effective elastic modulus 


$$
\frac{\partial \bar{\sigma}}{\partial \bar{\epsilon}}=\left(\frac{1}{N} \sum_{i=1}^{N} \frac{1}{E_{i}}\right)^{-1}
$$

Comparison of (4.8) with the stability condition (4.7) shows that the configuration with $l=1$ is stable if and only if the overall modulus of the whole chain is negative

$$
\frac{\partial \bar{\sigma}}{\partial \bar{\epsilon}}<0
$$

For the tri-linear springs (2.7), the condition (4.9) can be simplified further

$$
t>1-N^{-1}
$$

which can also be rewritten as

$$
t>t^{*}
$$

This inequality suggests that for $t<t^{*}$, all configurations with $l \geq 1$ are unstable. The critical value $t^{*}$ coincides with the one defined in (3.20) which allows us to conclude that condition (4.11) is identical to the condition distinguishing the regime of supercritical bifurcations.
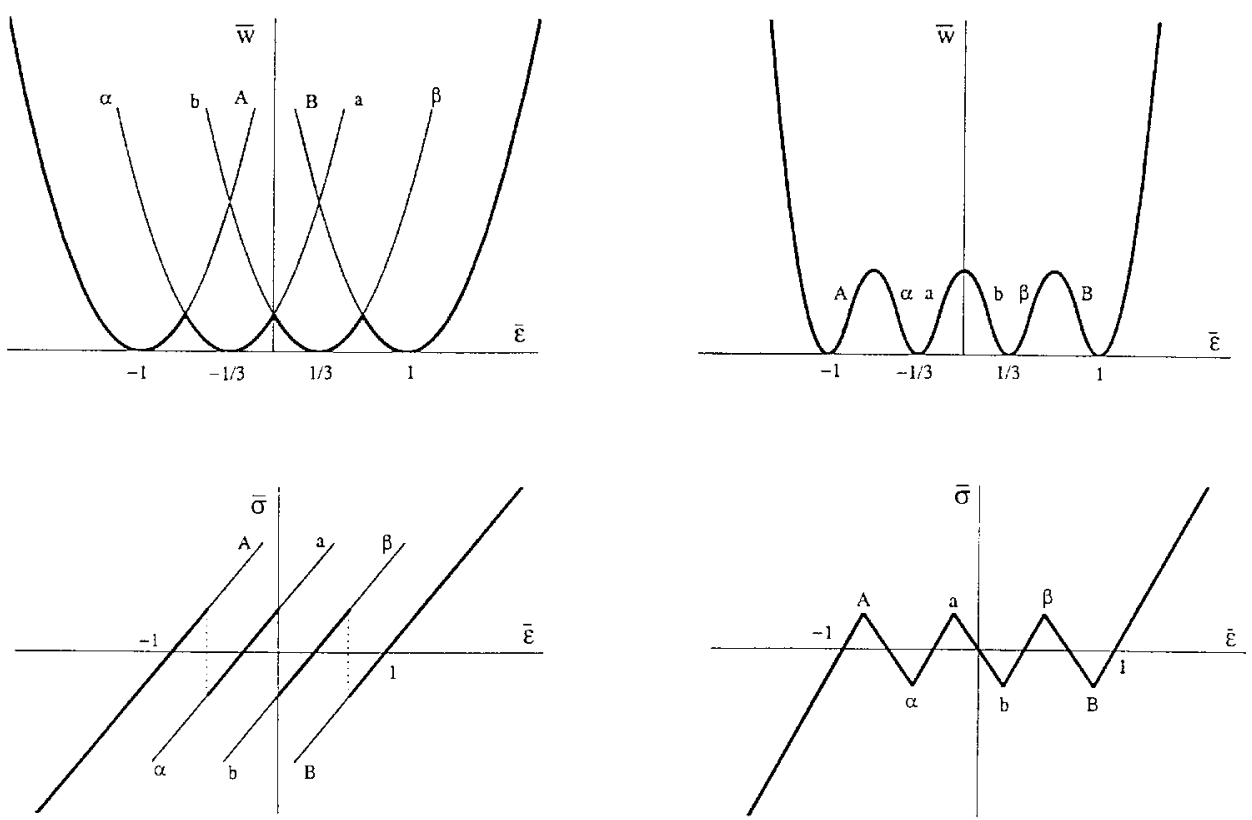

Fig. 13. Absolute minimizers and metastable states for the chain with three springs $(N=3)$ in a hard device: (a) subcritical, (b) supercritical cases. Absolute minima are shown with bold lines, metastable states with solid lines. Points $A, B, \alpha, \beta, a, b$ have the same meaning as in Fig. 7. 


\subsection{Hard device: illustrations}

To illustrate the difference in stability properties between the supercritical and the subcritical regimes, consider the chain with three tri-linear elements $(N=3)$. According to the criterion (4.11) the tetragonal branches $(2,1,0),(0,1,2)$ and the orthorhombic branch $(1,1,1)$ are metastable for $t>t^{*}=\frac{2}{3}$ and are unstable for $t<t^{*}=\frac{2}{3}$. The analysis for Fig. 13 shows that in the supercritical case $t>\frac{2}{3}$ all metastable configurations are also globally stable. On the contrary, for $t<\frac{2}{3}$ the bifurcations connecting globally stable branches are subcritical and there exist metastable configurations that are not global minimizers with a possibility of hysteresis in a quasistatic loading.

For a chain with three cubic springs (1.6) the metastable branches are $(3,0,0)$, $(0,0,3)$-cubic and $(2,0,1),(1,0,2)$-tetragonal. The following branches are unstable: $(0,3,0)$-cubic, and $(1,2,0),(0,2,1)$-tetragonal. The tetragonal branches $(2,1,0)$ and $(0,1,2)$ contain stable segments and the degenerate orthorhombic branch $(1,1,1)$ is neutrally stable. The hysteresis is again possible as in the case of subcritical tri-linear springs (see Fig. 6).

In the case of tri-linear springs and general $N$, the subcriticality condition $t<t^{*}$ distinguishes the case when all the branches with $l=1$ are unstable. The typical
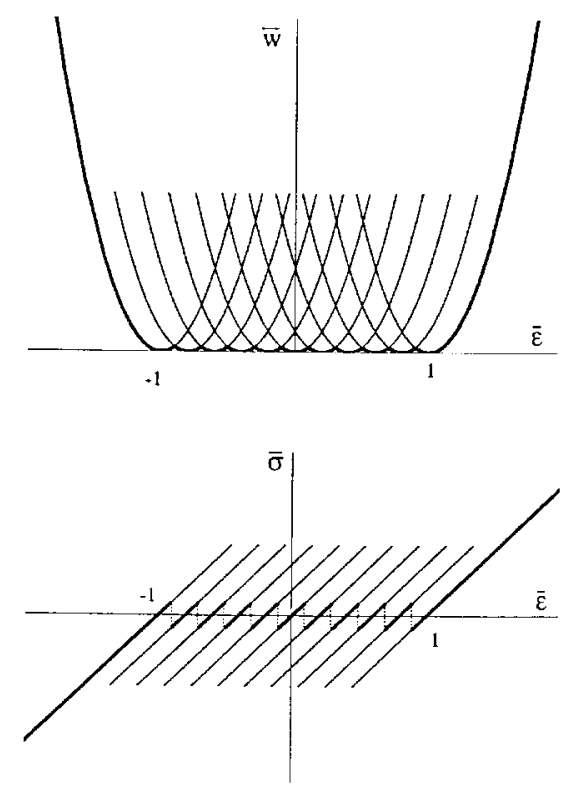

(a)
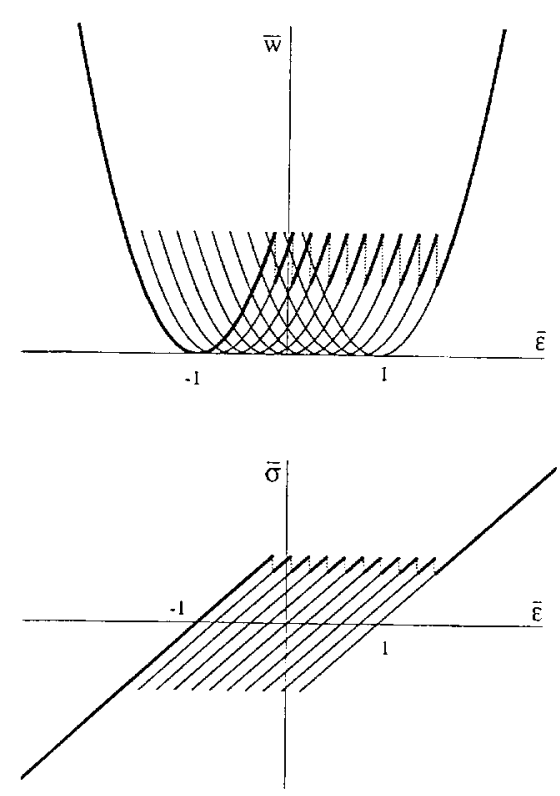

(b)

Fig. 14. Absolute minimizers and metastable states for the chain with ten tri-linear springs $(N=10)$ in a hard device: (a) 'Maxwell' path; (b) 'maximum hysteresis' or 'barrier free' path for increasing total displacement. 
picture for the overall behavior of the chain in this case is represented in Fig. 14, where we show both the hysteresis-free Maxwell path and the 'maximum quasistatic hysteresis' path, which utilizes local minima and does not involve any barrier crossing (Puglisi and Truskinovsky, 1998).

\subsection{Hard device: non-symmetric energy}

Here we also briefly comment on another interesting case of the tri-linear springs with non-equal elastic moduli of the stable phases. In this case a mixed subcritical-supercritical behavior can be observed. In fact, consider the following energy function of a single spring

$$
w(\epsilon)=\left\{\begin{array}{l}
\frac{1}{2}(\epsilon+1)^{2}, \quad \epsilon<-t_{1} \\
\frac{1}{2}\left(\gamma \epsilon^{2}+\lambda \epsilon+\eta\right), \quad-t_{1} \leq \epsilon \leq t_{2}, \\
\frac{1}{2} \delta(\epsilon-1)^{2}, \quad t_{2}<\epsilon
\end{array}\right.
$$

where parameters $t_{1}, t_{2}, \delta, \gamma, \eta$ and $\lambda$ are chosen in such a way that the energy function is smooth, for instance

$$
t_{2}=\frac{\delta-1+t_{1}(\delta+1)}{\delta+1+t_{1}(\delta-1)} .
$$

The explicit solution of the equilibrium equations can be obtained similarly to the case of symmetric energy (2.6). The typical overall energy-strain and the forcestrain relations are illustrated in Fig. 15 where only the metastable configurations are presented. Notice that the system behaves as in the subcritical case $\left(t<t^{*}\right)$ on part of the domain and as in the supercritical case $\left(t>t^{*}\right)$ on the other part of the domain: the energy has multiple local minima in one case and only the absolute minimum in the other. The mechanical response therefore changes with
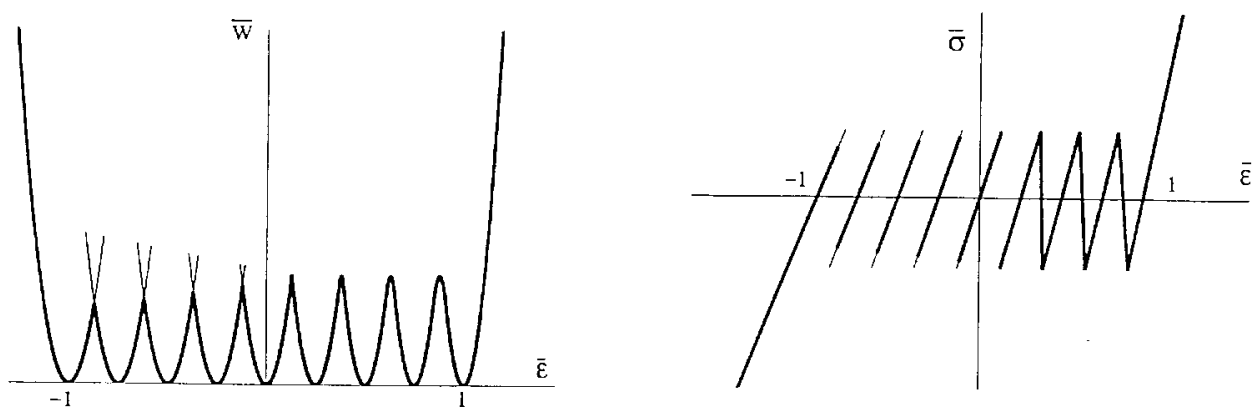

Fig. 15. Absolute minimizers and metastable states for the chain in hard device with six $(N=6)$ trilinear springs characterized by nonsymmetric energy wells. Absolute minima are shown with bold lines, metastable states with solid lines. 
the total strain and the hysteretic behavior may coexist with the reversible Maxwell behavior.

\subsection{Soft device: general case}

To study the local stability of the equilibria in the case of a fixed applied load, we rewrite the energy function as

$$
G\left(\epsilon_{1}, \ldots \epsilon_{N-1}\right)=W\left(\epsilon_{1}, \ldots \epsilon_{N}\right)-\bar{\sigma} \sum_{i=1}^{N} \epsilon_{i} .
$$

Since all variables are independent, the corresponding Hessian matrix takes the form
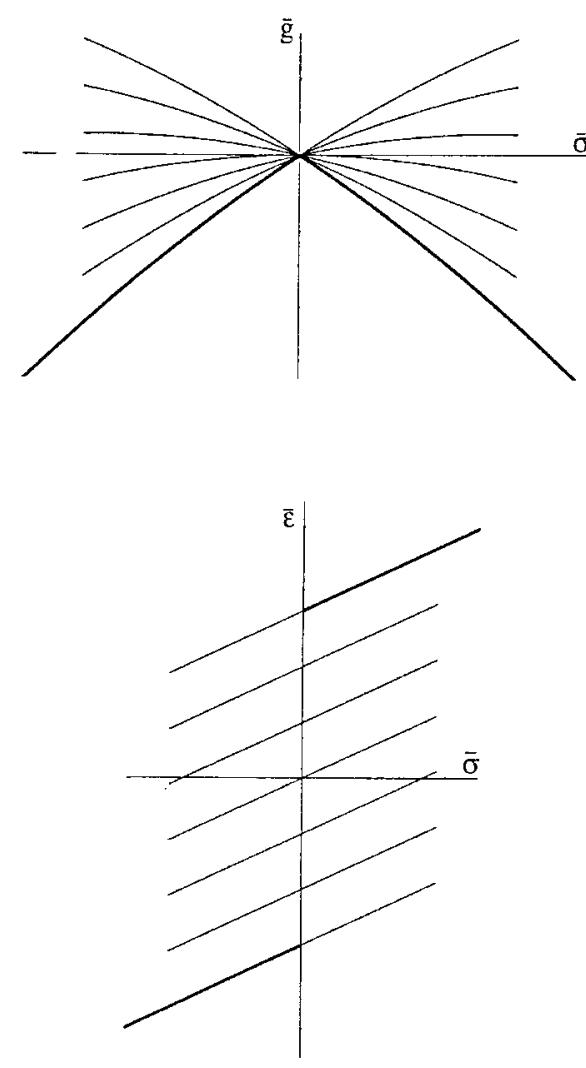

(a)
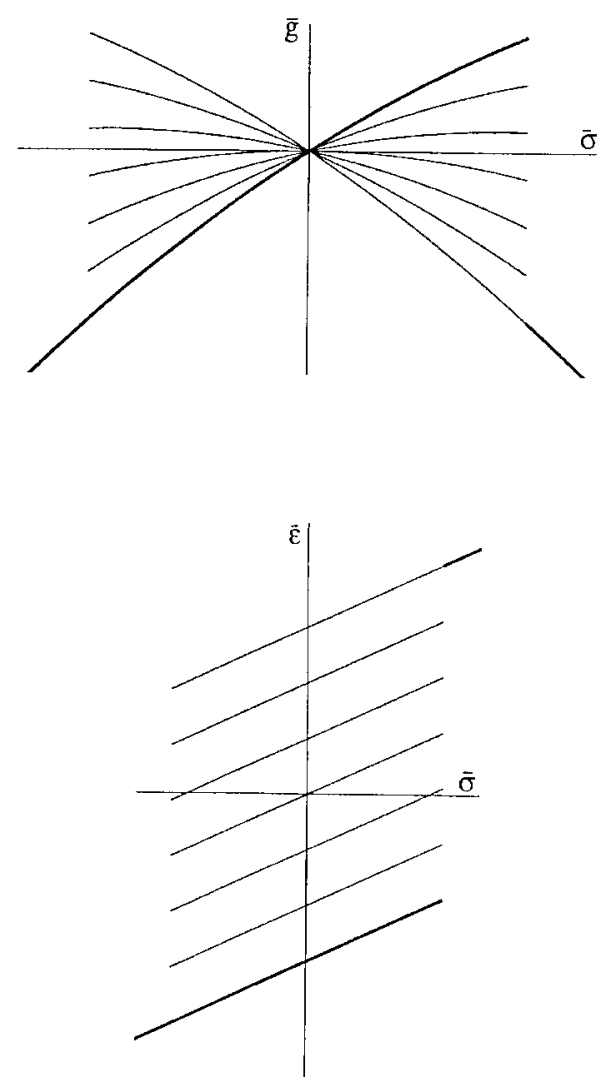

(b)

Fig. 16. Absolute minimizers and metastable states for the chain with six tri-linear springs $(N=6)$ in a soft device: (a) 'Maxwell' path and; (b) 'maximum hysteresis' path for an increasing total stress. 


$$
\mathbf{H}=\left|\begin{array}{llll}
E_{1} & 0 & \ldots & 0 \\
0 & E_{2} & \ldots & 0 \\
\ldots & \ldots & \ldots & \ldots \\
0 & 0 & \ldots & E_{N}
\end{array}\right|,
$$

where the entries $E_{i}=w^{\prime \prime}\left(\epsilon_{i}\right)$ are calculated for the equilibrium solution $\epsilon_{i}(\bar{\sigma})$. The diagonal matrix $\mathbf{H}(\bar{\sigma})$ is positive definite if and only if

$$
E_{i}>0, \quad i=1, \ldots, N .
$$

This condition excludes configurations with springs in the spinodal region $(l=0)$. As a result, the only metastable configurations in the soft device are the mixtures of stable phases $(k, 0, m)$. We remark that in the corresponding continuum problem studied by Ericksen (1975), the only metastable configurations are the Cauchy-Born ones. To illustrate the role of the local minima for the chain in a soft device, we present in Fig. 16 the picture of the overall behavior for the chain with $N=6$. Two limiting quasistatic paths are shown; the Maxwell path and the 'maximum hysteresis' paths, notice that both paths involve trivial (Cauchy-Born) configurations only.

\section{Discussion}

In this paper we studied a role of the spinodal region in the hysteretic behavior of a discrete chain with bi-stable elements. By varying the magnitude of the negative modulus in the spinodal region, we observed two different kinds of constitutive behavior.

In the case of steep force-elongation curve in the spinodal region, the energy landscape is characterized by multiple local minima and a number of paths are available to the system in quasistatic evolution. As the springs change phase the whole chain undergoes a sequence of subcritical bifurcations and the size of the hysteresis is independent of the number of springs. When the force-elongation curve in the spinodal region is sufficiently gentle, the energy landscape is different, all local minima are also global and no hysteresis is possible despite the nonconvexity of the energy. In this case all bifurcations are supercritical and the system shows stable softening behavior.

An important open question, which is outside the scope of purely static analysis, concerns the actual strategies for the switching between the local minima as the loading parameter changes. An understanding of this issue will lead to a better understanding of the rate-independent hysteresis associated with the underlying discreteness. In a separate paper (Puglisi and Truskinovsky, 1998) we investigate how well the discrete model reproduces important paradigms of the phenomenological Preisach model like 'return point memory' and 'congruency', which must be present in any realistic modeling of hysteresis.

Although the behavior of continuum models with nonconvex energies has been studied rather thoroughly, the relation of these models to their discrete prototypes 
remains basically unexplored. As the comparison of Ericksen's and MullerVillagio's models indicate, this relation is highly nontrivial at least in the prediction of the variety of local minima. Since it is the configurations of the local minima that determine the structure of the hysteresis, the formal extension of the nonconvexity of the elastic energy from discrete into continuum level is unsatisfactory. Therefore, the most nontrivial open question concerns the rigorous homogenized description of the chain in the limit when the number of springs goes to infinity. It is already clear that the corresponding limit has little to do with the straightforward long-wave approximation of the discrete problem and that the limiting theory will be a variant of a plasticity theory.

\section{Acknowledgements}

The work of L.T. was supported by the NSF grant DMS-9501433, the work of G.P. was supported by COFIN-MURST98 "Modelli Matematici per la Scienza dei Materiali".

\section{References}

Alinger, T.L., Epstein, M., Herzog, W., 1996. Stability of muscle fibers on the descending limb of the force-length relation. J. Biomechanics 5, 627-633.

Braides, A., Dal, Maso G., Garroni, A. 1998 Variational formulation of softening phenomenon in fracture mechanics. Preprint.

Ericksen, J.L., 1975. Equilibrium of bars. J. Elast. 5, 191-201.

Ericksen, J.L., 1984. The Cauchy and Born hypothesis for crystals. In: Gurtin, M. (Ed.), Phase transformations and material instabilities in solids. Academic Press, Orlando.

Ericksen, J.L., 1993. Local bifurcation theory for thermoelastic Bravais lattices. In: Microstructure and phase transitions, Lecture Notes in Physics, vol. 344. Springer, Berlin.

Fedelich, B., Zanzotto, G., 1992. Hysteresis in Discrete Systems of Possibly Interacting Elements with a Double-Well Energy. J. Nonlinear Sci. 2, 319-342.

Muller, I., Seelecke, S. 1996 Thermodynamic aspects of shape memory alloys. Preprint.

Muller, I., Villaggio, P., 1977. A model for an elastic-plastic body. Arch. Rat. Mech. Anal. 65, 25-46.

Puglisi, G., Truskinovsky, L., 1997. First and Second order Phase Transitions in a Discrete System with Bistable Elements. In: Proceedings of the XIII AIMETA Congress, Sienna, pp. 140-152.

Puglisi, G., Truskinovsky, L. 1998 Hysteresis in a chain with bi-stable elements. To be submitted.

Rogers, R., Truskinovsky, L., 1997. Discretization and hysteresis. Physica B 233, 370-375.

Truskinovsky, L., 1996. Fracture as a phase transition. In: Batra, R.C., Beatty, M.F. (Eds.), Contemporary research in the mechanics and mathematics of materials. A collection of papers dedicated to the 70th birthday of J.L. Ericksen. CIMNE, Barcelona, pp. 322-332.

Vainshtein, A., Healey, T., Rosakis, P., Truskinovsky, L., 1998. The role of the spinodal in one-dimensional phase transitions microstructures. Physica D 115, 29-48. 